\title{
Diacronie
}

Studi di Storia Contemporanea

$\mathrm{N}^{\circ} 15,3 \mid 2013$

Spazi, percorsi e memorie

\section{Turismo di guerra, turismo di pace: sguardi incrociati su Italia e Francia}

\section{Elisa Tizzoni}

\section{(2) OpenEdition \\ 1 Journals}

\section{Edizione digitale}

URL: http://journals.openedition.org/diacronie/430

DOI: 10.4000/diacronie.430

ISSN: 2038-0925

Editore

Association culturelle Diacronie

Notizia bibliografica digitale

Elisa Tizzoni, «Turismo di guerra, turismo di pace: sguardi incrociati su Italia e Francia », Diacronie [Online], $N^{\circ} 15,3$ | 2013, documento 3, online dal 01 octobre 2013, consultato il 10 décembre 2020. URL : http://journals.openedition.org/diacronie/430 ; DOI : https://doi.org/10.4000/diacronie.430 


\title{
Diacronie
}

\section{3/}

\section{Turismo di guerra, turismo di pace: sguardi incrociati su Italia e Francia}

\author{
Elisa TIZZONI*
}

L'articolo offre alcune riflessioni sul rapporto del turismo con i concetti di guerra $e$ pace in occasione dei due conflitti mondiali, analizzando in chiave comparata il contesto francese e quello italiano. Dopo una sintetica introduzione circa le principali ripercussioni dei conflitti contemporanei sul turismo e sui caratteri che esso può assumere in contesti di guerra, l'attenzione viene rivolta al fenomeno del cosiddetto "turismo di guerra", sviluppatosi nella fase finale e negli anni immediatamente successivi alla Grande Guerra, per poi esaminare il ruolo del "turismo di pace" all'interno del processo di ricostruzione di un ordine pacifico internazionale seguito alla fine del secondo conflitto mondiale.

\section{Introduzione}

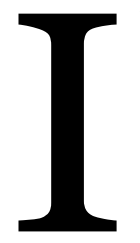

1 turismo rappresenta un fenomeno che, nel corso del Novecento, è andato assumendo dimensioni globali, conquistando, seppur solamente negli ultimi decenni, l'attenzione degli storici interessati alla ricostruzione dei caratteri dello sviluppo economico e dell'evoluzione dei consumi nel mondo contemporaneo, senza escludere i pur meno numerosi studi focalizzati sugli aspetti istituzionali delle politiche turistiche ${ }^{1}$.

${ }^{1}$ BATTILANI, Patrizia, Vacanze di pochi vacanze di tutti. L'evoluzione del turismo europeo, Bologna, Il Mulino, 2003; BERRINO, Annunziata, Storia del turismo in Italia, Bologna, Il Mulino, 2011; PALOSCIA, Franco, Storia del turismo nell'economia italiana, Città di Castello, Petruzzi, 1994; BOYER, Marc, Histoire générale du tourisme du XVIe au XXIe siècle, Paris, L'Harmattan, 2005; WALTON, John, «Prospects in tourism history: evolution, state of play and future developments», in Tourism Management, 30, 6/2009, pp. 783-793; LÖFGREN, Orvar, In Holiday. A History of Vacationing, Berkeley, Los Angeles-London, University of California Press, 1999. 
Tra i fenomeni più interessanti connessi alla diffusione della pratica della vacanza fra fasce di popolazione sempre più ampia, il cosiddetto turismo di guerra conseguente al primo conflitto mondiale, rappresenta un oggetto di studi particolarmente stimolante ${ }^{2}$.

L'espressione "turismo di guerra", specie se applicata al contesto del primo conflitto mondiale, può destare perplessità, considerando gli ostacoli che un evento bellico pone all'esercizio delle attività connesse alla vacanza, impedendo, in primis, quella libera circolazione che ne rappresenta il presupposto essenziale; le devastazioni materiali conseguenti alle guerre, inoltre, rischiano di compromettere la dotazione di infrastrutture e beni culturali delle destinazioni al punto da far perdere loro, talvolta definitivamente, la posizione conquistata nel mercato.

Ciononostante, il turismo di guerra acquisì un'importanza chiave per il rilancio dell'economia europea in occasione delle due guerre mondiali, qualificandosi nello stesso tempo come un fenomeno culturale e politico che condensava i sentimenti collettivi, le aspirazioni nazionali, il dolore e le speranze della società europea coinvolta nel conflitto.

Nel presente contributo, dopo un sintetico cenno alle principali ripercussioni dei conflitti contemporanei sul turismo e sulle possibili funzioni assolte da tale settore all'interno di questi, verranno proposte alcune riflessioni sulla posizione del turismo rispetto agli opposti concetti di guerra e pace in occasione dei due conflitti mondiali, partendo dall'analisi delle modalità e dei contenuti con i quali si sviluppò un'offerta specificamente dedicata alla visita dei campi di battaglia già durante le fasi finali della Grande Guerra, sia su iniziativa degli operatori economici privati che delle autorità pubbliche.

Successivamente, si tratterà del ruolo del turismo all'interno del processo di ricostruzione di un ordine internazionale pacifico dopo la Seconda guerra mondiale, valutando le differenze in termini ideologici ed organizzativi rispetto al contesto del primo dopoguerra.

La ricerca si soffermerà sul contesto italiano, inquadrandolo tuttavia nel più ampio panorama europeo e compiendo alcune comparazioni con le iniziative turistiche attuate

\footnotetext{
2 BRANDT, Susanne, «Le voyage aux champs de bataille», in Vingtième Siècle. Revue d'histoire, 41, 1994, pp. 18-22; JANSEN-VERBEKE, Myriam, GEORGE, Wanda, Reflections on the Great War Centenary: from Warscapes to Memoryscapes in 100 years, in BUTLER, Richard, WANTANEE, Suntikul (editors), Tourism and War. A Complex Relationship, London-New York, Routledge, pp. 273-287; LLOYD, David W., Battlefield Tourism. Pilgrimage and the Commemoration of the Great War in Britain, Australia and Canada, 1919-1939, Oxford, Berg, 1998; TREVES, Anna Lisa, Anni di guerra, anni di svolta: il turismo italiano durante la prima guerra mondiale, in BOTTA, Giorgio (a cura di), Studi geografici sul paesaggio, Milano, Cisalpino-Goliardica, 1989, pp. 250-299.
} 
in Francia sia in riferimento alla promozione dei luoghi simbolo della Grande Guerra, sia riguardo alla nascita di un nuovo approccio nei confronti del turismo, inteso quale strumento di pace, già a partire dagli anni Trenta.

1. «A beauty quite unknown and unsuspected»: il turismo e la Grande Guerra

Allo scoppio della Grande Guerra, in Europa, il turismo aveva raggiunto un considerevole grado di sviluppo, almeno nelle upper classes, dopo alcuni decenni di gestazione nel corso dei quali si era andato distinguendo da pratiche che ne rappresentano antecedenti storici, ma presentavano natura intimamente diversa, come quelle della villeggiatura o del viaggio per motivi di studio e formazione sul modello del Grand tour3.

Lo stato di guerra condizionò negativamente il settore turistico non solo a livello europeo, ma su scala globale e sotto molteplici aspetti, come ben sintetizzato nelle pagine de L'industria del forestiero in Italia, uno dei più noti testi di tipo manualistico dedicato agli operatori del settore, scritto da Angelo Mariotti nel $1923^{4}$, nel quale i principali ostacoli alla pratica turistica sono ricondotti all'inasprirsi dei controlli sui forestieri in ingresso a causa del timore di eventuali spie nemiche; alla limitata efficienza dei collegamenti ferroviari conseguente alla penuria di carbone e alla precedenza accordata ai convogli militari; alle requisizioni e sequestri nei confronti degli esercizi alberghieri collocati nelle zone alpine a ridosso del confine con l'Impero austro-ungarico, dove il turismo aveva assunto un ruolo fondamentale nell'economia locale.

Queste difficoltà materiali incisero in maniera drammatica sul settore, mandando in crisi non solo gli esercizi ristorativo-ricettivi e di fornitura di servizi collocati nelle aree di guerra ma, più in generale, tutto il sistema della mediazione fra domanda e offerta, comprendendo il comparto dei trasporti e delle agenzie di viaggio5.

3 TOWNER, John, An historical geography of recreation and tourism in the western world 1540-1940, Chichester, John Wiley \& Sons Ltd, 1996; TISSOT, Laurent, Industrie touristique, Tourist Industry. Construction d'une industrie touristique aux 19e et 20e siècles. Perspectives internationales, Development of a Tourist Industry in the 19th and 2oth Centuries. International Perspectives, Neuchâtel, Editions Alphil, 2000; CAVALCANTI, Maria Luisa, Riflessioni sulle vacanze degli italiani nella belle époque, in ISTITUTO PER LA STORIA DEL RISORGIMENTO ITALIANO - COMITATO DI NAPOLI (a cura di), Storia del turismo. Annale 2003, Milano, FrancoAngeli, 2004, pp. 55-68.

4 MARIOTTI, Angelo, L'industria del forestiero in Italia, Bologna, Zanichelli, 1923.

5 VISENTIN, Claudio (a cura di), Il turismo contemporaneo. Cultura e mondo dellimpresa, Lugano, Giampiero Casagrande editore, 2002. 
Le perdite maggiori furono imputabili alla cessazione dei flussi provenienti dall'America del Nord, colti dal panico sin dai primi venti di guerra, come testimoniato nelle pagine del «New York Times» dove si narra come, nell'estate del 1914, un vero e proprio «rush of tourists» stava abbandonando in tutta fretta l'Europa verso i porti degli Stati Uniti e del Canada, attraverso uno scalo in Inghilterra ${ }^{6}$.

Anche le destinazioni italiane, nonostante l'iniziale non belligeranza, erano considerate pericolose dai visitatori stranieri; le stesse iniziative del Governo per facilitare l'evacuazione dei viaggiatori accrescevano la preoccupazione tra essi, poiché venivano interpretate come un segnale della prossima entrata in guerra dell'Italia7.

Sin dai primi mesi del conflitto, il declino dei flussi turistici fu rapido e netto, come rilevato da uno studio commissionato nella seconda metà del 1915 dalla Bibliothèque universelle et revue Suisse in riferimento al mercato elvetico, secondo il quale le entrate derivanti dal turismo nel 1914 erano calate del 40\%; nella città di Lucerna, ad esempio, nell'agosto del 1913 gli arrivi avevano sfiorato le 74.000 unità, mentre l'anno successivo il loro numero si ridusse a poco più di $8.000^{8}$.

L'assenza forzata dei turisti dalle mete europee, tuttavia, rafforzò la loro attrattività, nonostante la diffusione delle notizie riguardanti le pesanti distruzioni perpetrate ai danni del patrimonio architettonico e alle infrastrutture. Lo sviluppo del turismo di guerra che, come vedremo, mosse i primi passi a conflitto ancora aperto dipese, tuttavia, soprattutto dagli effetti della propaganda a sostegno prima dell'intervento e poi della continuazione del conflitto posta in atto tanto da parte degli Imperi centrali che delle potenze dell'Intesa ${ }^{9}$.

La ricerca di consenso all'entrata in guerra e successivamente - man mano che le privazioni subite dalla popolazione civile si intensificavano e cresceva il numero dei caduti al fronte - il bisogno di stimolare costantemente nell'opinione pubblica l'adesione alle motivazioni ideologiche e morali che le autorità pubbliche adducevano per giustificare la partecipazione al conflitto, condusse

\footnotetext{
${ }^{6}$ S. a., «England meeting rush of tourists», in New York Times, 25 agosto 1914, p. 4. 7 Ibidem.

8 S. a., «Giornali e Riviste», in La Stampa, 10 dicembre 1915, p. 3.

9 ROSSINI, Daniela, La propaganda nella Grande guerra tra nazionalismi e internazionalismi, Milano, Unicopli, 2007; ISNENGHI, Mario, Il mito della Grande guerra, Bologna, Il Mulino, 2002; MASAU DAN, Maria, PORCEDDA, Donatella, (a cura di), L'arma della persuasione: parole ed immagini di propaganda nella Grande guerra, Mariano del Friuli, Edizioni della Laguna \& Cooperativa Mitt, 1991; ALEXANDRE, Arsene, GINISTY, Paul, Le livre du souvenir: guide du voyageur dans la France envahie en 1914; HORNE, John, "Corps, lieux et nation. La France et l'invasion de 1914», in Annales Histoire, Sciences Sociales, 55, 1/2000, pp. 73-109; PROST, Antoine, WINTER, Jay, Penser la grande guerre: un essai d'historiographie, Paris, Seuil, 2004.
} 
abbastanza spontaneamente quasi tutti i giornali - e non necessariamente e non soltanto perché costretti dall'intervento degli uffici di censura predisposti dalle autorità militari e politiche - ad agire come organi politicamente responsabili degli effetti che possono produrre e quindi a funzionare come articolazioni del sistema di guerra $^{10}$.

La guerra assumeva una dimensione mitica ed estetizzata sia nella comunicazione pubblica elaborata dai movimenti nazionalisti, dove si esaltava la forza rigeneratrice del conflitto, che avrebbe stimolato il rinnovamento della "vecchia" società europea valorizzando le energie ed il coraggio delle giovani generazioni, sia in quella delle forze politiche e intellettuali di sinistra, che in essa vedevano l'occasione per rovesciare gli equilibri sociali, politici ed economici consolidati.

Questo approccio al conflitto fece breccia nell'opinione pubblica degli Stati Uniti, dai quali provenivano i flussi turistici di maggior interesse per le destinazioni europee, portatori di moneta pregiata e interessati ad un vasto paniere di beni e servizi e fu corroborata tramite campagne informative propagandistiche apparse sui maggiori quotidiani. Queste descrivevano l'azione militare contro le potenza della Triplice alleanza nei termini di uno scontro di civiltà, che avrebbe deciso della sopravvivenza dei valori della civiltà europea attaccata dai nuovi barbari raccolti sotto le insegne della Prussia e dei suoi alleati ${ }^{11}$.

Immersi in quest'atmosfera, i corrispondenti statunitensi descrissero i campi di battaglia con gli stessi toni utilizzati da molte delle guide turistiche contemporanee in riferimento alle più affascinanti mete europee, cogliendo gli elementi esteticamente ed emotivamente appetibili dei luoghi, seppure oggetto delle devastazioni.

Un esempio concreto di questi orientamenti è offerto da un esteso articolo di Whitney Warren, noto architetto newyorkese, corrispondente del «New York Times» dal fronte italiano, dove i concetti chiave legati al portato emozionale della permanenza sui campi di battaglia riprendono proprio quei motivi, prodotti dalla propaganda interventista, che nel dopoguerra sarebbero stati tradotti in un'offerta turistica destinata in particolare al mercato americano: a partire dal concetto della guerra come spettacolo e dei campi di battaglia come "teatri" sui quali questo veniva rappresentato.

10 ISNENGHI, Mario, La Grande Guerra, Firenze, Giunti, 1993.

${ }^{11}$ KINGSBURY, Celia, Malone, Food Will Win the War, For Home and Country: World War I Propaganda on the Home Front, Lincoln, University of Nebraska Press, 2010; BUITENHUIS, Peter, The Great War of Words. British, American and Canadian Propaganda and Fiction, 1914-1933, Vancouver, University of British Columbia Press, 1987; ROSSINI, Daniela, Woodrow Wilson and the American Myth in Italy: Culture, Diplomacy, and War Propaganda, Cambridge, Harvard University Press, 2008. 
Warren dichiara infatti di essersi imbattuto in "a beauty quite unknown and unsuspected. I speak of the spectacle of the War and for once not of Art or of Nature» ${ }^{12}$.

Le pagine di Warren si soffermano sulle glorie passate dell'Italia, alle quali tuttavia si accosta un presente nel quale il coraggio e la potenza bellica trovavano la loro affermazione, in un'ideale continuità segnata dalla grandezza del "genio" italiano:

It is a popular idea, but a false one, that the past is better than the present [...] it is impossible to forget the scenery in which this prodigious events are taking place. [...] Italy is a great modern country that is writing at this moment the most splendid pages of her history. She may well compare herself today with the Italy of past centuries ${ }^{13}$.

Anche oltralpe la promozione del turismo occupò le prime pagine dei maggiori quotidiani francesi rivolti ai visitatori provenienti dai paesi alleati e neutrali:

Le comité de propagande touristique à l'étranger, fondé en 1915 par le TouringClub de France, s'attache a faire connaitre aux alliés et aux neutres les richesses naturelles et artistiques de la France, ses stations thermales et leur valeur curative. Il lutte contre la propagande des Austro-Allemands dont l'activité est plus intense que jamais ${ }^{14}$.

Nel 1916 il Conseil supérieur du tourisme francese era già all'opera per programmare le iniziative da attuarsi per rilanciare il settore una volta terminate le ostilità, annunciando l'obbiettivo di «faciliter à nos amis étrangers la visite des champs de bataille où s'illustrent nos armées et des autres sites de la France pittoresque», concentrandosi su «les régions où la guerre aura laissé un souvenir historique» ${ }^{15}$.

Meno di un anno dopo, l'Ufficio nazionale del turismo francese organizzò un vero e proprio press tour sui campi di battaglia de l'Ourcq e della Marna a beneficio di un gruppo di giornalisti provenienti da diverse nazioni, affinché questi potessero promuovere il fascino dei luoghi della guerra avendone avuto esperienza diretta ${ }^{16}$.

12 WARREN, Whitney, «Italy in the War Is a Wonderful Spectacle», in New York Times, 12 novembre 1916, p. 2.

13 Ibidem.

${ }^{14}$ S. a., "Communiqués», in Le Figaro, 31 maggio 1917, p. 3.

15 S. a., «Information», in Le Figaro, 30 ottobre 1916, p. 4; la stessa notizia è riportata con i medesimi contenuti in S.a., "Une réunion du Conseil supérieur du tourisme», in L'Humanitè, 28 ottobre 1916.

${ }^{16}$ S. a., «Au champ de bataille de l'Ourcq», in Le Figaro, 28 settembre 1917, p. 2; l'articolo citato non specifica nè il numero, nè la provenienza dei giornalisti. 
Le politiche turistiche durante gli anni del conflitto subirono, dunque, una sospensione parziale piuttosto che una vera e propria cessazione, cosicché la ripresa poté realizzarsi precocemente e con rapidità.

Come riconosciuto efficacemente nel già citato studio di Mariotti, motivazioni psicologiche, riassumibili in un comune "senso di sollievo", motivazioni politiche, riconducibili alla visita degli ex combattenti ai campi di battaglia e motivazioni economiche, legate alla svalutazione della lira, stimolarono la rinascita del turismo italiano, non diversamente da quanto avvenne in altre aree europee, individuando nel turismo di guerra un settore trainante ${ }^{17}$.

La piena ripresa della corrente turistica tra Stati Uniti ed Europa fu tuttavia rallentata dalle restrizioni ai viaggi oltreoceano imposte dalle autorità nordamericane, in gran parte ancora in vigore nella seconda metà del 1919, e dalla carenza di vettori di trasporto, poiché la flotta passeggeri a disposizione dei cittadini statunitensi era stata sensibilmente ridotta sia a causa della guerra sottomarina che della riconversione di molti navigli a cargo mercantili ${ }^{18}$.

Nonostante le restrizioni al trasporto di passeggeri, unite alla penuria di merci e alle difficoltà nel ripristino dei più elementari servizi al viaggiatore, i primi turisti, inizialmente organizzati per necessità in gruppi, ripresero a visitare l'Europa partendo proprio dai luoghi teatro di guerra.

Il numero annuale di turisti provenienti dagli Stati Uniti, pari a circa 246.00o nel 1913 e poi precipitato a 65.000 nel 1915 e a 25.000 nel $1917^{19}$, risalì a 91.000 già nel 1919, ritornando ai livelli prebellici nell'anno successivo, con un totale di 196.000 visitatori responsabili di una spesa di 101 milioni di dollari (162 milioni comprendendo i costi per il trasporto versati tramite le agenzie di viaggio del paese di provenienza) ${ }^{20}$.

L'esaltazione del Vecchio continente, funzionale a giustificare l'impegno bellico statunitense a difesa dell'Europa, ne aveva accentuato il fascino, mentre tassi di cambio favorevoli costituivano ulteriori incentivi al viaggio, stimolando le iniziative degli operatori della mediazione turistica: nella primavera del 1919 Gilbert E. Fuller, Presidente dell'American Association of Tourist and Ticket Agents, effettuò un soggiorno di tre mesi in Europa proprio allo scopo di stringere intese commerciali in vista di una piena ripresa dei flussi turistici, con particolare attenzione a Inghilterra, Belgio, Francia e Italia.

${ }_{17}$ MARIOTTI, Angelo, op. cit., p. 11.

18 Ibidem.

$19 \quad$ Revue d'economie politique, 39, 1925, p. 18.

20 ANDERLA, M. George, «Le tourisme international en Europe», in Etudes et conjoncture Economie mondiale, 4, 1950. pp. 21-58. 
Esponendo i risultati della missione commerciale, Fuller sottolineava innanzitutto l'opportunità di pianificare la visita ai teatri di guerra entro l'anno successivo, prima che gli interventi di ricostruzione cancellassero i segni del conflitto.

Tra le zone di maggior interesse turistico Fuller includeva l’Italia, sostenendo:

the italians expect their visitors to show an interest in the sections of Tirol and the Dolomites which will be Italian, and to pay more attention to the italian lakes"

Poco oltre si aggiunge che le più interessanti attrazioni sarebbero consistite in:

districts showing the horror of war; cities which were practically under constant shellfire and must be rebuilt; districts showing the desolation of war; districts showing the difficulties under which armies fought ${ }^{21}$.

La prima comitiva turistica statunitense giunse in Europa il 2 ottobre 1919 con il transatlantico Mauretania, facente parte della flotta dell'American express travel Department: composta da poche decine di privilegiati, essa comprendeva autorevoli personalità tra le quali un Senatore e un membro del Congresso ${ }^{22}$.

Le richieste dei turisti interessati alle destinazioni europee si fecero via via più intense, nonostante gli alti costi di viaggio imputabili al permanere di difficoltà dovute ai danni subiti dalle infrastrutture dei paesi belligeranti, alla lentezza nell'eliminazione delle limitazioni alla libera circolazione, alla scarsità di viveri e generi di prima necessità, alla conversione di gran parte delle navi da trasporto passeggeri a quello delle merci.

La promozione del turismo di guerra avvenne non solo attraverso i canali comunicativi ampiamente sperimentati nei decenni precedenti, ma anche ricorrendo a nuove forma di politiche turistiche adeguate ai cambiamenti irreversibili generati dalla guerra sul mercato turistico e, più in generale, sulla società occidentale.

I governi europei, infatti, consapevoli del fatto che l'arrivo di flussi di visitatori da oltreconfine avrebbe potuto contribuire significativamente alla ripresa economica, iniziarono a considerare il turismo - la cui promozione, nei decenni precedenti la guerra era essenzialmente delegata all'iniziativa di operatori privati e autonomie locali - come un settore di diretto intervento delle autorità centrali, non più limitato alla sola

${ }^{21}$ U. S. OFFICIAL PHOTO-SIGNAL CORPS U.S.A, «Outlook for touring in Europe next autumn», in New York Times, 18 maggio 1919, p. 84.

${ }^{22}$ LEVICK, Edwin, «Pleasure tours to Europe possible, but difficult», in New York Times, 19 ottobre 1919, p. 3. 
definizione del quadro normativo generale entro il quale esercitare le attività connesse alla vacanza, ma consistente nell'adozione di vere e proprie politiche per la valorizzazione delle destinazioni ${ }^{23}$.

Seguendo l'esempio della Francia, dove già nel 1910 era stato istituito l'Office national du tourisme, nella maggior parte dei paesi europei vennero creati degli Uffici nazionali per il turismo, che coordinassero e curassero direttamente una vasta gamma di attività, tra le quali la supervisione su una rete di punti d’informazione per i viaggiatori siti sul territorio nazionale e all'estero; la stipula di accordi e convenzioni per ottenere facilitazioni su trasporti e servizi per i visitatori stranieri; la raccolta di dati e la realizzazione di studi.

In Italia la promozione del turismo di guerra fu pertanto affidata soprattutto all'attività dell'Ente nazionale industrie turistiche (ENIT), costituito nel 1919, il quale, con la collaborazione della la Compagnia italiana turismo (CIT) e del Touring Club (TCI) nel 1921 celebrò i luoghi legati alla Grande curando una pubblicazione denominata Visite ai Campi di battaglia, che suscitò il plauso degli esperti del settore:

merita speciale menzione l'opuscolo delle Visite ai campi di battaglia, contenente sedici itinerari automobilistici attraverso le zone più famose e più gloriose della nostra guerra. Con questo opuscolo l'Enit ha assolto anche un dovere di patriottismo, invitando i turisti a compiere un pellegrinaggio che è al tempo stesso un rito solenne e che vale a dare un'idea delle enormi difficoltà che i soldati italiani dovettero superare per strappare all'agguerrito nemico la completa vittoria ${ }^{24}$.

L'annessione del Trentino-Alto Adige, peraltro, si rivelò un evento particolarmente favorevole per il sistema turistico italiano, poiché, specialmente nell'area del Sud Tirolo, lo sviluppo del turismo alpino verificatosi nei decenni precedenti ${ }^{25}$ aveva stimolato la realizzazione di strutture ricettive e complementari di alto livello, finanziate con capitali provenienti da Austria, Germania, Svizzera e in buona parte gestite da operatori con elevata professionalità formatisi in questi stessi paesi.

In seguito alla Conferenza di Parigi, le strutture alberghiere di proprietà straniera situate nella Venezia tridentina vennero confiscate e trasferite a proprietari italiani,

\footnotetext{
23 BATTILANI, Patrizia, op. cit., p. 32.

24 MARIOTTI, Angelo, op. cit., pp. 35-36.

25 BOYER, Marc, «Les Alpes et le tourisme», in Histoire des Alpes, 9, 2004, pp. 19-30; TISSOT, Laurent, From Alpine tourism to the "Alpinization" of tourism, in ZUELOW, Eric (editor), Touring Beyond the Nation, Farnham, Ashgate, 2011, pp. 59-78.
} 
riprendendo rapidamente l'attività, cosicché durante l'estate del 1920 in quest'area furono registrati circa 100.000 arrivi $^{26}$.

Come testimoniato dal proliferare di guide turistiche per la visita ai campi di battaglia $^{27}$, durante gli anni del fascismo, il turismo di guerra fu ulteriormente incentivato, celebrando il conflitto non solo come teatro del patriottismo e dell'eroismo nazionale, ma anche e soprattutto come il momento storico che aveva segnato la rinascita della nazione, compiutamente realizzata dal regime ${ }^{28}$.

Anche nella celebre serie «Le cento città d'Italia illustrate», che ebbe una vastissima diffusione grazie al carattere divulgativo (pur in presenza di testi scritti da autorevoli pubblicisti) e al prezzo popolare di ciascun fascicolo, pubblicata con cadenza settimanale tra il 1924 e il 1929 dalla casa editrice milanese Sonzogno, venne dedicato ampio spazio ai campi di battaglia.

Nell'opuscolo delle zone di Bassano-Monte Grappa leggiamo:

Da Bassano si scorge e sovrasta il monte sacro alla Patria [...] Monte Grappa, che tutti gli italiani dovrebbero visitare con religioso raccoglimento. [...] Ancora il visitatore di questa montagna trova ovunque i segni delle lotte gigantesche che hanno fatto del Grappa il grande simbolo della intangibilità della Patria29.

Simili accenti ricorrono anche nelle pagine che descrivono l'altopiano di Asiago:

Asiago, risorta ora dalle rovine più bella, più linda, più ridente, guarda con fiducia all'avvenire e custodisce i grandi ricordi e i monti gloriosi coi quarantuno cimiteri di guerra sparsi nel suo territorio, e volle dedicato alla Brigata Regina il suo Parco, e all'eroico generale Turba il cimitero militare ${ }^{30}$.

In maniera ancora più esplicita, l'opuscolo su Udine attribuisce alla città l'appellativo di "Capitale della guerra", sottolineando, nelle conclusioni, che «la capitale

${ }^{26}$ MARIOTTI, Angelo, op. cit., p. 32.

${ }^{27}$ Solo per citare alcune opere: TOURING CLUB ITALIANO, Sui campi di battaglia: guida storico-turistica, Milano, Touring Club Italiano, 1928; ENTE NAZIONALE PER LE INDUSTRIE TURISTICHE, I campi della gloria: itinerario illustrato delle zone monumentali dei campi di battaglia da Trieste a Trento, Roma, ENIT, 1927.

${ }^{28}$ GENTILE, Emilio, La Grande Italia: The Rise and Fall of the Myth of the Nation in the Twentieth Century, Madison, University of Wisconsin Press, 2009.

29 S. a., Bassano e il Monte Grappa, in Le cento città d'Italia illustrate, fasc. 18, Milano, Sonzogno, pp. 15-16.

$3^{\circ}$ S. a., Vicenza e l'altipiano di Asiago, in Le cento città d'Italia illustrate, fasc. 17, Milano, Sonzogno, p. 16. 
della guerra è ora la capitale di un più esteso Friuli ai confini della Patria: memore sentinella del sacrificio, cara ad ogni cuore di italiano» ${ }^{3}$.

La visita ai sacrari dei caduti in combattimento assumeva valenze politiche oltre che sentimentali e culturali: il sacrificio dei soldati nel primo conflitto mondiale non sarebbe valso solamente a preservare la storia e la civiltà europee ma avrebbe costituito la base per rifondarne la società.

Le opere architettoniche ed artistiche realizzate per commemorare i caduti e testimoniare ai posteri le vicende del conflitto cercavano dunque di rendere tangibile la continuità tra passato e futuro saldata dall'esperienza bellica; in particolar modo il cimitero divenne «the vehicle for making the past present, for affording a vision of the essence of Italian identity, for inspiring future action» 32 .

Questi aspetti sono evidenti nell'opuscolo de «Le cento città d'Italia» dedicato ad Aquileia, dove, in conclusione del largo spazio che descrive il «Cimitero degli eroi», l'autore scrive:

ed a chi riguarda in qualunque ora del giorno, il verde cupo delle piante, la mole secolare della basilica, le tombe fiorite con allineati, vigili giganti, i cipressi, sembra invero di immergersi nella millenaria nostra civiltà attingendovi non il senso della morte, ma il senso della vita33.

Poco oltre si aggiunge:

Ed ora rinnoviamo l'invito che ci ha indotti a scrivere queste pagine: vadano gli italiani ad Aquileia. Prima della guerra, l'Austria [...] mandava ogni anno in gita d'istruzione gli alunni delle sue scuole superiori. Altrettanto stiamo facendo noi pure, ma non nella misura e con l'intensità necessarie: occorre conoscere meglio, per meglio conoscere noi stessi, questa austera figlia di Roma34.

Tanto le diverse condizioni socio-economiche che la storia politica, dunque, marcarono la differenza tra il carattere assunto dalla visita ai campi di battaglia nel corso degli anni Venti in Italia e negli altri paesi europei: in Francia e Belgio si registrò

${ }^{31}$ CHINO, Ermacora, Udine. La capitale della guerra, in Le cento città d'Italia illustrate, fasc. 75, Milano, Sonzogno, p. 16.

${ }^{2}$ BURDETT, Charles, Journeys through Fascism. Italian Travel Writing Between the Wars, Oxford, Berghahn Books, 2007, p. 96.

33 CHINO, Ermacora, Aquileia. La figlia di Roma, in Le cento città d'Italia illustrate, fasc. 76, Milano, Sonzogno, p. 14 .

34 Ibidem, p. 16. 
soprattutto l'afflusso di turisti stranieri, inizialmente a maggioranza inglese, ai quali si aggiunsero successivamente i tedeschi, protagonisti di quello che era percepito non tanto e non solo come un viaggio di piacere, ma come un vero e proprio pellegrinaggio35, sebbene diversi osservatori, per lo più appartenenti alle fila della Sinistra, criticassero questo fenomeno ritenendolo un «mercantilisme scandaleux» della memoria nazionale 36 .

In Italia l'afflusso di stranieri presso i luoghi della guerra fu parzialmente compromesso dal basso livello qualitativo delle strutture e delle infrastrutture nazionali; negli anni Venti, inoltre, in presenza di un basso livello dei consumi che impediva il consolidarsi di una significativa domanda turistica interna, i luoghi del conflitto divennero soprattutto meta di escursioni giornaliere, spesso organizzate da associazioni dopolavoristiche e istituzioni pubbliche, promosse nell'ambito di quella "educazione" ai valori patriottici forgiata dal regime con l'obbiettivo ultimo di cementare il consenso verso di esso 37.

\section{2. «Un grande fattore di pace e di conoscenza fra i popoli». Il turismo e la ricostruzione di un ordine mondiale pacifico nel secondo dopoguerra}

Sin dal 1945, analogamente a quanto già verificatosi al termine della Grande Guerra, l'impegno del settore privato in favore di una pronta rinascita del turismo europeo fu sostenuto dall'organizzazione turistica nazionale, considerando che l'arrivo di flussi stranieri, nordamericani in particolare, avrebbe rappresentato una delle poche voci in attivo delle bilance commerciali delle nazioni appena uscite dal conflitto; parallelamente, si cercò di sviluppare le potenzialità del turismo come fenomeno culturale e sociale destinato a contribuire alla ricostruzione di un ordine mondiale pacifico.

Occorre ricordare che la maggiore estensione dei territori direttamente interessati da distruzioni e rastrellamenti e la maggiore intensità delle devastazioni, attuate con bombardamenti aerei, resero più arduo il ripristino delle dotazioni turistiche e del sistema delle comunicazioni rispetto al 1918.

La ripresa dell'attività degli organismi per il coordinamento e la promozione fu tuttavia rapida: già nel 1947 l'International Union of Official Tourist Publicity Organisations (IUOTPO) venne rifondato, assumendo una nuova denominazione

\footnotetext{
35 BRANDT, Susanne, op. cit.

${ }^{6} 6$ SNELL, Victor, «Pélerine», in L'Humanité, 26 luglio 1919, p. 2.

37 DE GRAZIA, Victoria, The Culture of Consent, Cambridge, Cambridge University Press, 1981.
} 
(International Union of Official Travel Organisations, IUOTO) e un assetto maggiormente strutturato rispetto al passato ${ }^{38}$.

In Italia ciò fu ancora più precoce: già nell'inverno del 1945 si tenne a Milano il convegno degli Enti provinciali per il turismo (EPT) dell'Alta Italia, nel corso del quale si delinearono le possibili linee guida del percorso di normalizzazione e ricostruzione del settore.

In questa occasione alle eventuali obiezioni da parte degli osservatori contemporanei circa l'opportunità di occuparsi del tema del turismo in un'Italia segnata dalle ferite fisiche e morali della guerra e in una condizione istituzionale ed economica di profonda emergenza, uno dei personaggi chiave per il settore dell'accoglienza nella prima metà del Novecento, Giuseppe Sommariva ${ }^{39}$, rispondeva definendolo come «fattore di quella ricostruzione verso la quale tendono gli sforzi di tutti i volenterosi italiani animati dal proposito di arrivare ad un'auspicata normalizzazione»40.

In quello che fu dunque un momento di fermento operativo e di elaborazione teorica sulla natura del fenomeno turistico all'interno di una società europea che sarebbe stata rifondata all'insegna di nuovi valori e nuovi stili di vita, la collocazione del settore rispetto ai poli concettuali opposti di pace e guerra variò nettamente rispetto all'epoca della Grande Guerra: mentre nel primo caso, come osservato nei paragrafi precedenti, si assistette all'affermazione del turismo di guerra, nel secondo se ne diffuse una visione come strumento di pace.

Nel caso dell'Italia e della Francia, ma le stesse riflessioni sarebbero applicabili ad altri paesi, un vero e proprio turismo di guerra comprendente la visita ai luoghi simbolo della Seconda guerra mondiale e, soprattutto, della Resistenza, si sarebbe affermato solamente più tardi, a seguito di un lungo e doloroso processo di elaborazione di uno dei periodi più tragici e controversi della storia di queste due nazioni, e successivamente alla nascita, a partire dagli anni Novanta, dei primi complessi museali

${ }^{8} 8$ Negli anni Settanta, la IUOTO è divenuta parte della rete delle agenzie ONU, trasformandosi nella World Tourism Organization (WTO) attiva ancora oggi.

$39 \mathrm{Su}$ questo personaggio, che importò in Italia il modello europeo dell'agenzia di viaggi, è stato scritto che «lo sosteneva [...] una visione del turismo di ampio respiro, nella quale tutti i diversi settori - e quindi lo Stato, con leggi opportune, le amministrazioni ferroviarie, l'industria alberghiera, le stazioni climatiche e termali ecc. - avrebbero dovuto svilupparsi involontaria ma stretta coordinazione, in vista dell'interesse comune». VISENTIN, Claudio, op. cit., p. 78.

40 Studi e Proposte per una riforma della legislazione turistica nazionale. Convegno degli Enti Provinciali per il Turismo dell'Alta Italia (Milano 7-8 novembre 1945), Bergamo, Stamperia Conti S.A., 1945, p. 4. 
ed espositivi svincolati da una logica esclusivamente commemorativa e aperti a funzioni didattiche e di edutainement ${ }^{41}$.

Dopo il 1945, invece, giunse a piena maturazione l'elaborazione teorica sul turismo come fenomeno "umanitario e sociale", prodotta da associazioni internazionali, tecnici ed esperti del settore nei decenni precedenti.

M. Pierre Audigier, presidente della Fédérations des syndicats d'initiative de France, Colonies et Protectorats, nel Rapport sur l'Activité du Tourisme Réceptif et la Situation actuelle du Tourisme Français, pubblicato alla metà degli anni Trenta, aveva definito il turismo «vrai patriotisme à la fois humain et social [...] patriotisme de paix et de progrès» 42 .

Con gli stessi accenti, il saluto ufficiale recato dal Ministro dei Lavori pubblici francese al Conseil central du tourisme international tenutosi a Parigi nel maggio 1934 si era concluso con questo auspicio: «Je crois fermement que vous arriverez à faire tomber un certain nombre des barrières qui existent entre les peuples et que vous collaborerez ainsi à l'affermissement de la paix»43.

Nel corso degli anni Trenta, persino nelle pagine di un giornale di orientamento nazionalista e antidemocratico come il francese «Le Matin» erano state riportate le dichiarazioni del Direttore del Centre national d'expansion du tourisme francese ${ }^{44}$, Dulaurens, su quanto la rinascita del turismo favorisse la conoscenza reciproca dei popoli: «les échanges touristiques restent le meilleur moyen pour les peuples de s'apprécier et de se connaître»45.

Coerentemente con questa linea di pensiero, nel citato convegno degli Enti provinciali per il turismo dell'Alta Italia tenutosi nel 1945, Leonetto Gamerra, responsabile dell'EPT di Bergamo, si lanciava in un invito di carattere generale:

è fondamentale mostrare una mentalità ospitale, nella consapevolezza del valore del patrimonio turistico italiano non solo passato ma anche soprattutto futuro. È la nostra continuità che dobbiamo far manifesta e vorrei quasi dire la nostra eternità, l'indistruttibilità di un impero che nessun trattato e nessuna guerra vittoriosa poté

${ }^{41}$ VIEREGG, Hildegard, Rappresentare la storia contemporanea nei musei, in DI VALERIO, Franca, PATICCHIA, Vito (a cura di), Un futuro per il passato. Memoria e musei nel terzo millennio, Bologna, Clueb, 2000, pp. 131-15.

42 FÉDÉRATIONS DES SYNDICATS D'INITIATIVE DE FRANCE, COLONIES ET PROTECTORATS, CONSEIL CENTRAL DI TOURISME INTERNATIONAL, Bulletin officiel des Fédérations des syndicats d'initiative de France, Colonies et Protectorats, 4/1935, p. 133.

43 ID., Bulletin officiel des Fédérations des syndicats d'initiative de France, Colonies et Protectorats, 4/1934, p. 106.

44 Il "Centre national d'expansion du tourisme francese" fu fondato nel 1935 e liquidato nel 1941. 45 S.a., «Thermalisme et hôtellerie vont renaître. La France doit rester le jardin de l'Europe», in Le Matin, 25 giugno 1941, p. 1. 
mai darci, ma che nessun trattato e nessuna guerra infelice potrà mai toglierci. L'unico impero adatto al nostro carattere, alla nostra mentalità, alla nostra storia, alla nostra civiltà: un impero ideale che è veramente il più vero, il più vasto, il più solido impero del mondo ${ }^{46}$.

Quest'approccio al turismo fu sostenuto anche in occasione del primo congresso nazionale del turismo, tenutosi a Genova nel 1947, primo grande evento per gli addetti al settore nell'Italia repubblicana; nella relazione introduttiva ai lavori del convegno, tenuta da Umberto Faruffini, rappresentante dell'EPT di Milano, si auspicava la piena ripresa del turismo «per la sua natura permeata di gentilezza», affinché esso potesse contribuire «a ristabilire nel mondo nei nostri confronti quella conciliazione quella simpatia delle quali abbiamo estremo bisogno»47.

Tre anni dopo il convegno di Genova, nell'ambito del primo congresso nazionale del Gruppo parlamentare per il turismo, tenutosi nel 1950 a Trento e Bolzano -capoluoghi di un'area, quella del Trentino Alto Adige, che vantava una consolidata presenza nei mercati turistici internazionali - fu affermato che «il turismo sociale sul piano internazionale è un grande fattore di pace e di conoscenza fra i popoli» 48 .

Negli stessi anni, nella maggior parte dei paesi europei furono dedicate notevoli risorse alla promozione del cosiddetto turismo sociale, orientato non solo a rispondere a bisogni di tipo ricreativo e salutistico delle classi lavoratrici, ma anche a stimolare la conoscenza reciproca e la tolleranza fra i popoli tramite soggiorni di scambio e altre iniziative simili49.

Anche in questo caso si trattava del compimento di un percorso che era iniziato nei decenni precedenti, poiché già nel XIX secolo si erano andate diffondendo teorizzazioni sociologiche che suggerivano di rendere disponibili alle classi lavoratrici attività ricreative finanziate e organizzate dallo Stato, al fine di tutelarne l'equilibrio psico-

\footnotetext{
${ }^{46}$ Studi e Proposte, cit., p. 33.

47 CAMERA DI COMMERCIO INDUSTRIA E AGRICOLTURA DI GENOVA, Problemi del turismo: memorie e note presentate al primo congresso nazionale del turismo (Genova, 15-19 maggio 1947), Genova, Pagano, 1947, p. 20.

48 GRUPPO PARLAMENTARE PER IL TURISMO, Atti del Primo Congresso Nazionale del Gruppo Parlamentare per il Turismo (Trento-Bolzano, 10-13 settembre 1950), s.l., s.e., p. 55 . 49DACHARRY, Monique, «Le tourisme social», in Revue de géographie alpine, 53, 4/1965, pp. 633-641; MINNAERT, Lynn, DIEKMANN, Anya, McCABE, Scott, Defining Social Tourism and Its Historical Context, in ID. (eds.), Social tourisme in Europe. Theory and practice, BristolTonawanda-Nort York, Channel View, 2012, pp. 18-30; DALL'ARA, Giancarlo, Turismo sociale, storia e attualità del diritto alle vacanze, Rimini, Panozzo editore, 2005.
} 
fisico ed evitare che spendessero il tempo libero in attività contrarie alla morale pubblica50.

L'esperienza del conflitto e il diffuso desiderio di un ritorno ad una pace stabile e duratura che ne seguì, ampliarono obbiettivi e aspirazioni delle politiche a sostegno di una migliore fruizione del tempo libero dei lavoratori: non si trattava più di ritemprare fisico e mente del lavoratore aumentando la sua efficienza sul posto di lavoro o offrendo un più attivo e collaborativo sostenitore al regime; la vacanza era entrata nel novero dei bisogni del cittadino, costituendo un'esperienza fondamentale per la sua formazione e la sua coscienza civica.

Nello stesso tempo, dopo la fine della Seconda guerra mondiale l'ulteriore sviluppo di movimenti dei lavoratori di ispirazione internazionalistica aveva creato nelle classi popolari il necessario retroterra culturale per stimolare il desiderio di conoscere nuove culture tramite l'esperienza della vacanza51.

L'esiguità dei mezzi finanziari resi disponibili per tali iniziative limitò tuttavia la diffusione di viaggi di scambio internazionali a lungo raggio tra le classi popolari. Tuttavia, anche grazie a istituti quali il gemellaggio e alla nascita di associazioni internazionali per la promozione del turismo sociale e giovanile, il turismo poté effettivamente contribuire a stimolare la conoscenza fra i popoli, almeno tra quelli europei ${ }^{2}$.

\section{Conclusioni}

L'esame delle fonti citate nei paragrafi precedenti indica che, mentre dal punto di vista economico gli obbiettivi di fondo ai quali fu indirizzata la ricostruzione del sistema turistico dopo la Prima e la Seconda guerra mondiale furono sostanzialmente gli stessi, mirando al rapido ripristino trasporti del sistema turistico così da attirare i flussi americani portatori di moneta forte, dal punto di vista ideologico sono riscontrabili notevoli differenze.

\footnotetext{
$5^{0}$ In molti casi furono direttamente i datori di lavoro privati, le grandi fabbriche soprattutto, che si fecero carico, anche economicamente, delle attività dopolavoristiche dei dipendenti, prevedendo positive ricadute relativamente alla produttività del lavoro.

${ }^{11}$ MAGISTRALI, Giuseppe, Turismo sociale, Sant'Arcangelo di Romagna, Maggioli, 2008. Nel 1963 nacque il Bureau international du tourisme social, struttura di confronto tra settore pubblico e privato, anch'essa attiva sino ai giorni nostri con la nuova denominazione di Social tourism organisation.

${ }^{2}$ WALTON, John K,, Understanding Social Tourism over Time and across Cultures: an International Historical Perspective, paper presentato al seminario NET-STaR su Social tourism and destination re generation, tenutosi a Bradford il 12 febbraio 2012, URL:

< http://www.westminster.ac.uk/_data/assets/pdf_file/ooog/129447/J-Walton-Sem3presentation.pdf $>$ [consultato il 31 luglio 2013].
} 
Durante la Grande Guerra, infatti, quando ancora le ostilità erano aperte, la valorizzazione turistica dei luoghi del conflitto si andò strettamente intrecciando alla propaganda esercitata sull'opinione pubblica europea ed extraeuropea per appoggiare la causa dei militari al fronte, dipingendoli come protagonisti di un epico scontro tra i valori della civiltà europea e la barbarie dei regimi autoritari aderenti alla Triplice alleanza.

Nel 1945, al contrario, risentendo d'idee già elaborate nel corso dei decenni precedenti, per le classi dirigenti europee «against the background of the traumatic depths of the recent conflict, the assertion that tourism could make a valuable contribution to peace making was highly credible»53.

Il turismo divenne un campo di attuazione di nuove forme di solidarietà internazionale e contribuì alla rinascita di un sistema di relazioni interstatali pacifico, frutto dell'incontro e della conoscenza, tra i popoli.

Una simile lettura risulta tuttavia troppo semplicistica, innanzitutto poiché la promozione del turismo di guerra dopo il 1918, pur facendo perno su contenuti elaborati nell'ambito della propaganda interventista, era parte di un programma di sviluppo turistico a lungo termine, all'interno del quale la visita ai campi di battaglia costituiva un'offerta rapidamente ripristinabile e facilmente spendibile sui mercati internazionali, che, si auspicava, avrebbe richiamato l'attenzione della domanda nella prospettiva del graduale ripristino del sistema turistico nel suo complesso:

\footnotetext{
Nos ruines, hélas! et nos champs de bataille ne seront pas que des pèlerinages, mais des buts d'excursion. [...] Profitons de la curiosité, que attirera chez nous cette affluence de visiteurs et de touristes, pour leur apprendre à aimer nos villes, nos champs et nos montagnes, et pour leur donner le gout d'y revenir54.
}

Parallelamente, come affermato in alcune delle relazioni tenutesi al convegno di Bergamo nel 1945, in maniera simile a quanto verificatosi dopo il primo conflitto mondiale, si affidava in parte la rinascita del turismo italiano al «flusso alimentato dagli ambienti degli eserciti d'oltreoceano che qui verranno indotti a ritornare,

53 HUGUES, George, Tourism, Sustainability, and Social Theory, in LEW, Alan A., HALL, C. Michael, WILLIAMS, Allan M. (eds.), A Companion to Tourism, Oxford, Blackwell Publishers, 2004, p. 498.

54 MEYAN, Paul, «Tourisme», in Le Figaro, 16 agosto 1916, p. 6. 
richiamati da nostalgici pensieri e dal culto dei loro morti che qui riposano in terra nostra»55.

Negli ultimi decenni il fenomeno del turismo di guerra ha assunto nuovi contenuti in sintonia con il diffondersi di una concezione esperienziale della vacanza, divenendo un turismo di memoria che considera la visita ai luoghi simbolo delle guerre mondiali e di altri conflitti non solo un viaggio di piacere, ma anche un pellegrinaggio attraverso la storia e le vicende personali delle popolazioni vittime dei combattimenti56.

Ancora oggi, tuttavia, sopravvivono forme di turismo di guerra interessate, talvolta con risvolti macabri e notevoli rischi per l'incolumità dei viaggiatori, a vivere le "emozioni" legate alla visita di luoghi politicamente instabili e interessati da combattimenti57. L'approccio adottato dalle organizzazioni internazionali del turismo in questi ultimi decenni ha invece rafforzato il concetto di turismo come strumento di pace e dialogo, declinandolo all'insegna dei principi di sostenibilità e responsabilità emersi in seguito al palesarsi degli effetti più distruttivi del turismo di massa ${ }^{8}$.

\footnotetext{
55 Studi e Proposte, cit., p. 366.

${ }^{56}$ ALESSANDRONE PERONA, Ersilia, Dai luoghi della memoria alla memoria di luoghi: $i$ Musei della Resistenza in Italia, Asti, Blu, 2005, pp. 187-197.

57 HALL, Charles Michael, DALLEN, J. Timothy, DUVAL, David Timothy, Safety and Security in Tourism: Relationships, Management, and Marketing, London, Routledge, 2003.

${ }^{58}$ LEVY, Stuart, HAWKINS, Donald, «Peace Through Tourism: Commerce Based Principles and Practices», in Journal of Business Ethics, 89, 4/2009, pp. 569-585; DE CARLO, Manuela, CASO, Raffaella, Focus on turismo e sostenibilità. Principi, strumenti, esperienze, Milano, Franco Angeli, 2007. Si veda l'articolo 3 dello Statuto del WTO, la cui missione è «the promotion and development of tourism with a view to contributing to economic development, international understanding, peace, prosperity and universal respect for, and observance of, human rights and fundamental freedoms».
} 


\section{* L'autore}

Elisa Tizzoni ha conseguito il Dottorato di ricerca in Storia Contemporanea presso l'Università di Pisa ed è attualmente cultore della materia presso l'Università di Firenze; i suoi interessi di ricerca riguardano principalmente i tourism studies, analizzati da un punto di vista istituzionale e culturale, le questioni relative al rapporto città-campagna e le dinamiche del processo di integrazione europea.

URL: < http://www.studistorici.com/progett/autori/\#Tizzoni >

\section{Per citare questo articolo:}

TIZZONI, Elisa, «Turismo di guerra, turismo di pace: sguardi incrociati su Italia e Francia», Diacronie. Studi di Storia Contemporanea : Spazi, percorsi e memorie, 29/10/2013,

URL:< http://www.studistorici.com/2013/10/29/tizzoni_numero_15/ >

Diacronie Studi di Storia Contemporanea www.diacronie.it

Risorsa digitale indipendente a carattere storiografico. Uscita trimestrale. redazione.diacronie@hotmail.it

Comitato di redazione: Marco Abram - Jacopo Bassi - Luca Bufarale - Alessandro Cattunar - Elisa Grandi - Deborah Paci - Fausto Pietrancosta - Matteo Tomasoni - Luca Zuccolo

Diritti: gli articoli di Diacronie. Studi di Storia Contemporanea sono pubblicati sotto licenza Creative Commons 2.5 . Possono essere riprodotti a patto di non modificarne i contenuti e di non usarli per fini commerciali. La citazione di estratti è comunque sempre autorizzata, nei limiti previsti dalla legge. 